\title{
Complete Spectrum of Multidepth Corrugated Circular Waveguides
}

\author{
Smain Amari, Rüdiger Vahldieck, Senior Member, IEEE, and Jens Bornemann, Senior Member, IEEE
}

\begin{abstract}
The paper presents a rigorous full-wave analysis of the complete spectrum of multidepth corrugated circular waveguides. The propagation constants are determined from the classical eigenvalues of a canonical matrix eigenvalue problem instead of a complex determinant. The method is used to determined the entire $k_{\mathrm{o}}-\boldsymbol{\beta}$ diagram of a dual-depth circular waveguide.
\end{abstract}

Index Terms - Eigenvalues and eigenfunctions, periodic structures, waveguide discontinuities.

\section{INTRODUCTION}

I NCREASING demand for high-power microwaves has led to the development of new devices in which corrugated waveguides play a major role [1], [2]. Successful design of these structures require an accurate analysis of the propagation properties of a corrugated waveguide in which the inner radius is a continuous function of the axial variable $z$.

The propagation properties of these periodic systems are commonly determined using expansions in space harmonics. The propagation constants of the Floquet modes are determined from a nonlinear determinant equation [1]. Such an approach can be time consuming, especially when a large number of modes is required. It also presents the risk of missing degenerate or closely packed roots of the determinant. The presence of complex modes in these structures poses an additional problem. An excellent discussion of the advantages of a classical matrix eigenvalue formulation over a determinant equation is given by Davies [3].

This letter proposes to show how an alternative formulation leading to a canonical matrix eigenvalue equation for the propagation constant of the Floquet modes can be developed. The method is based on an extension of the formulation presented in [4] where the unit cell of the periodic structure has only two well-defined discontinuities. This method is extended here to deal with the case of an arbitrary number of discontinuities per unit cell.

\section{THEORY}

Consider a unit cell of a periodically corrugated circular waveguide. The period of the structure is $p$ and there are $N$

Manuscript received September 16, 1998.

S. Amari and R. Vahldieck are with the Laboratory for Field Theory and Microwave Electronics, Swiss Federal Institute of Technology (ETH), Zürich, Switzerland.

J. Bornemann is with the Department of Electrical and Computer Engineering, University of Victoria, Victoria, B.C., Canada V8W 3P6.

Publisher Item Identifier S 1051-8207(99)02010-3. step discontinuities per unit cell. The case of sinusoidal corrugations can be approximated by step discontinuities whose radius is a sinusoidal function of $z$, for example.

The starting point of the formulation is to assume that the true transverse electric field at the discontinuities $1, \cdots, N+1$ are given by unknown functions $\boldsymbol{X}_{1}, \ldots, \boldsymbol{X}_{N+1}$. The Floquet condition is automatically satisfied by requiring that

$$
X_{N+1}=e^{-\gamma p} \boldsymbol{X}_{1} \text {. }
$$

Here, $\gamma$ is the propagation constant of the mode. Following the coupled-integral-equation technique (CIET), a set of $N$ coupled integral equations for the remaining $N$ unknown vector functions are derived [5]. To solve these integral equations, the functions $\boldsymbol{X}_{1}, \cdots, \boldsymbol{X}_{N}$ are expanded in series of suitable basis functions

$$
\boldsymbol{X}_{i}=\sum_{q=1}^{M} c_{q}^{(i)} \boldsymbol{Q}_{q}^{(i)}, \quad i=1, \cdots N .
$$

Substituting these expansions in the integral equations and applying the moment method [6], we get $N$ sets of linear equations in the coefficients $c_{q}^{(i)}$, namely

$$
\begin{aligned}
{[A]\left[c^{(1)}\right]+[B]\left[c^{(2)}\right]+e^{\gamma p}[C]\left[c^{(1)}\right] } & =0 \\
{[D]\left[c^{(1)}\right]+[E]\left[c^{(2)}\right]+[F]\left[c^{(3)}\right] } & =0 \\
\cdots+\cdots+\cdots & =0 \\
\cdots R]\left[c^{(N-2)}\right]+[S]\left[c^{(N-1)}\right]+[T]\left[c^{(N)}\right] & =0 \\
{[U]\left[c^{(N-1)}\right]+[V]\left[c^{(N)}\right]+e^{-\gamma p}[W]\left[c^{(N)}\right] } & =0 .
\end{aligned}
$$

The entries of the matrices in this equation involve sums over the modes of the sections; they can be inferred from the discussion in [5] and are not given here for lack of space.

The presence of two different functions of $\gamma$ in this equation prevents us from directly using a standard eigenvalue approach. To circumvent this problem, we first express the unknown vectors $\left[c^{(2)}\right]$ and $\left[c^{(N-1)}\right]$ in terms of the two vectors $\left[c^{(1)}\right]$ and $\left[c^{(N)}\right]$. This can be done using rows 2 to $N-1$ in (3). Using the resulting expressions of $\left[c^{(2)}\right]$ and $\left[c^{(N-1)}\right]$ in the first and last rows of (3), we get two equations in $\left[c^{(1)}\right]$ and $\left[c^{(N)}\right]$ of the form

$$
\left[\begin{array}{ll}
R_{1} & R_{2} \\
R_{3} & R_{4}
\end{array}\right]\left[\begin{array}{c}
c^{(1)} \\
c^{(N)}
\end{array}\right]+\left[\begin{array}{cc}
0 & R_{5} e^{\gamma p} \\
R_{6} e^{-\gamma p} & 0
\end{array}\right]\left[\begin{array}{c}
c^{(1)} \\
c^{(N)}
\end{array}\right]=0 .
$$

Using the notation $\lambda=e^{\gamma p}$, this equation can be transformed into the canonical form $[A][X]+\lambda[B][X]=0$. The details are not given here but can be found in [4]. 




Fig. 1. Side view of a dual-depth corrugated waveguide.

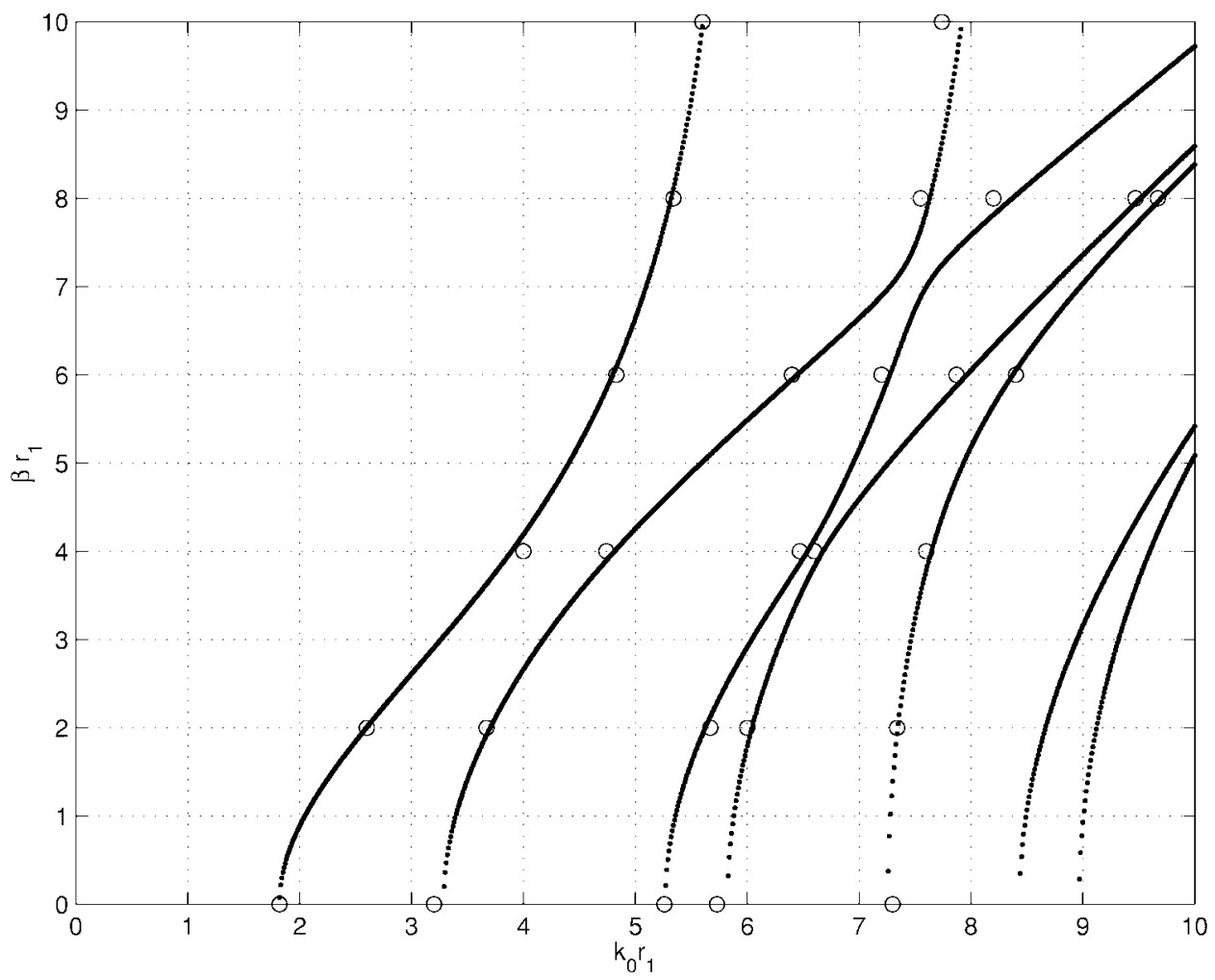

Fig. 2. Propagation constants of the first few propagating modes. The circles are from [7]

\section{APPLICATION TO DUAL-DEPTH CORRUGATED WAVEGUIDE}

The present approach was applied to determine the propagation constants in a dual-depth corrugated waveguide investigated in [7] (Fig. 1). In this case, the modes of the waveguide sections are used as basis functions. All the presented numerical results were obtained using four basis functions.

Fig. 2 shows the propagation constants of the first few branches in the dispersion diagram. Only modes with unity angular dependence are shown $[\cos (\phi)]$. The solid lines are from the present work and the circles are from [7]. It is evident that our calculations agree very well with the quoted results for the following dimensions: $r_{1}=40 \mathrm{~mm}, r_{01} / r_{02}=1.67, p=8$ $\mathrm{mm}, g=10 t=10 / 11 \mathrm{p}$, and $r_{01}=48.8 \mathrm{~mm}$.

A major advantage of the present approach is that it allows accurate analysis of complex, propagating, and evanescent modes simultaneously. Fig. 3 shows the real and imaginary 


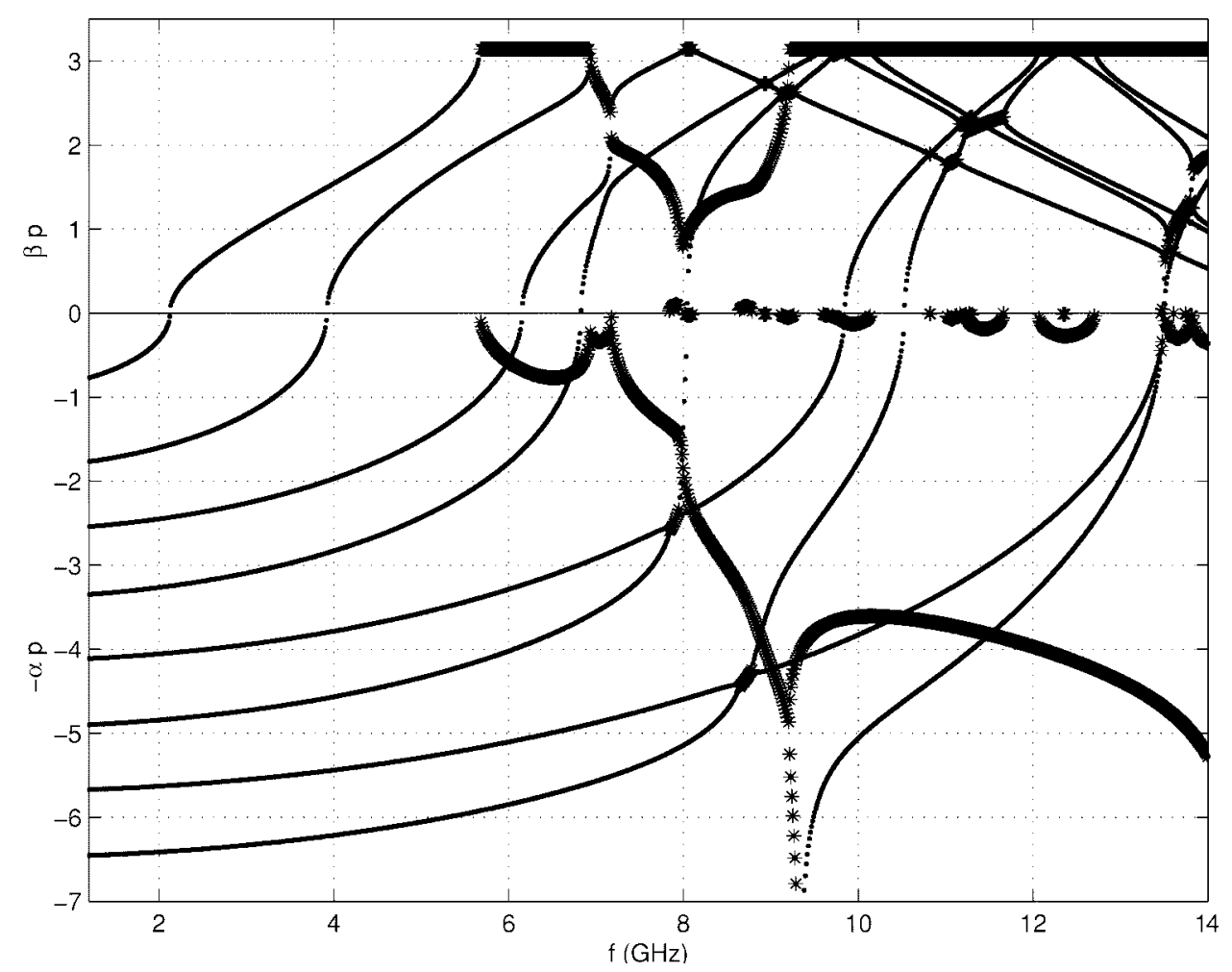

Fig. 3. Real and imaginary parts of propagation constant versus frequency. The stars represent complex modes. $r_{01}=1.22 r_{1}, r_{01}=1.13 r_{1}$, $p=0.5 r_{1}, g=10 t=10 / 11 \mathrm{p}$.

parts of the propagation constant as a function of frequency for the same dimensions as in Fig. 2. The stars represent complex modes. An interesting feature of the spectrum shown in Fig. 3 is the presence of modes with a constant value of $\beta p=\pi$. These modes are in fact present in simple periodic structures as shown by Collin [8, p. 367].

We finally mention that higher branches appear in Fig. 3 when more basis functions are used.

\section{CONCLUSIONS}

The approach presented in [4] is extended to deal with propagation in periodic structures in which the unit cell contains multiple discontinuities. Numerical results, obtained from our classical eigenvalue formulation, for a dual-depth corrugated circular waveguide are in excellent agreement with available data. Propagating, evanescent, and complex modes are determined simultaneously.

\section{REFERENCES}

[1] V. L. Granatstein and I. Alexeff, High-Power Microwave Sources. Boston, MA: Artech House, 1987.

[2] M. R. Amin, K. Ogura, H. Kitamura, K. Minami, T. Watanabe, Y. Carmel, W. Main, J. Weaver, W. W. Destler, and V. L. Granatstein, "Analysis of the electromagnetic waves in overmoded finite length slow wave structures," IEEE Trans. Microwave Theory Tech., vol. 43, pp. 815-822, Apr. 1995

[3] J. B. Davies, "Complete modes in uniform waveguides," pp. 8-10 in Finite-Element Software for Microwave Engineering, T. Itoh, G, Pelosi, and P. P. Silvester, Eds. New York: Wiley, 1996.

[4] S. Amari, R. Vahldieck, J. Bornemann, and P. Leuchtmann, "Propagation in circular waveguide periodically loaded with dielectric disks," in IEEE MTT-S Intern. Microwave Symp. Dig., Baltimore, MD, 1998, pp. $1143-1147$.

[5] S. Amari, J. Bornemann, and R. Vahldieck, "Accurate analysis of scattering from multiple waveguide discontinuities using the coupledintegral-equation technique," J. Electromagn. Waves Appl., vol. 10, pp. 1623-1644, Dec. 1996.

[6] R. F. Harrington, Field Computation by Moment Methods. Malabar, FL: Krieger, 1987.

[7] A. D. Olver, K. K. Yang, and P. J. B. Clarricoats, "Propagation and radiation behavior of dual-depth corrugated horns," Proc. Inst. Elec. Eng., vol. 131, pt. H, pp. 179-185, June 1984.

[8] R. E. Collin, Foundations for Microwave Engineering. New York: McGraw Hill, 1966. 